\title{
EDITORIAL
}

\section{Progress in novel cognitive enhancers for cognitive aging and Alzheimer's disease}

\author{
Diana W Shineman*, Aaron J Carman, Penny A Dacks, Rachel F Lane and Howard M Fillit
}

\begin{abstract}
Increased knowledge of the biology of synaptic function has led to the development of novel cognitive-enhancing therapeutic strategies with the potential for increased efficacy and safety. This editorial highlights a diverse array of approaches currently being explored to target cognitive dysfunction due to aging and/or Alzheimer's disease.
\end{abstract}

Keywords: Cognitive aging, Alzheimer's disease, Dementia, Cognitive enhancer, mGlu5, nAChR, M1 AChR, 5-HT6, Norepinepherine, CAMP

\section{Introduction}

Aging is the single most significant risk factor for Alzheimer's disease (AD). As we age, certain cognitive functions decline, a process known as cognitive aging. Cognitive function begins to decline in young adulthood, possibly as early as in the second or third decade of life. While there is ongoing debate as to how the trajectory of normal cognitive aging relates to the pathological progression of $\mathrm{AD}$, the development of novel cognitiveenhancing therapies is vital for improving function and quality of life for individuals suffering from cognitive impairment due to cognitive aging or AD.

Currently available US Food and Drug Administration (FDA)-approved drugs for AD either inhibit acetylcholinesterase (AChE) or target the $N$-methyl-D-aspartate receptor to improve cognitive function. Many other cognitive-enhancing strategies are under investigation. Caffeine, B vitamins and docosahexaenoic acid are examples of natural compounds that have been reported to improve cognition, although it is important to note that these agents can affect people in different ways and more scientific research is needed [1-3]. Exercise, meditation and other dietary factors have also been proposed to enhance cognition, although the data are far from conclusive $[3,4]$. Here, we highlight several exciting pharmacological therapeutic strategies that may both enhance cognitive function and offer potential to slow disease progression in $\mathrm{AD}$ patients. These strategies are

\footnotetext{
* Correspondence: DShineman@alzdiscovery.org

Alzheimer's Drug Discovery Foundation, 57 West $57^{\text {th }}$ Street, Suite 904 , New York, NY 10019, USA
}

explored in more detail in this special issue on 'Cognitive enhancers for aging and Alzheimer's disease'.

\section{Metabotropic glutamate receptor subtype 5}

Memory impairment may be due to an imbalance of inhibitory and excitatory neurotransmission. The excitatory neurotransmitter, glutamate, is decreased in the hippocampus of $\mathrm{AD}$ patients. Glutamate can modulate excitatory postsynaptic currents via the metabotropic glutamate receptor subtype 5 (mGlu5). Increasing the activation of mGlu5 may offer an exciting new therapeutic strategy to enhance cognitive function in AD patients. Jeff Conn's group at Vanderbilt University has developed a series of mGlu5 positive allosteric modulators for $\mathrm{AD}$, schizophrenia and other neurological conditions [5]. As opposed to direct activation of the mGlu5 receptor, positive allosteric modulators boost the response of this receptor to glutamate and enhance synaptic plasticity while avoiding unwanted side-effects seen with direct activation.

\section{Nicotinic acetylcholine receptor}

The neuronal nicotinic acetylcholine receptor (nAChR) is another important target for cognitive-enhancing therapies. The nAChRs are expressed in the hippocampus, a key brain area implicated in cognitive dysfunction with both aging and Alzheimer's disease. nAChRs function both presynaptically to regulate neurotransmitter release and postsynaptically where they can activate intracellular signaling cascades involved in learning and memory. Activation of the $\alpha 7 \mathrm{nAChR}$ subtype with small-molecule 
agonists enhances long-term potentiation and improves learning and memory across multiple cognitive domains in rodents, monkeys, and humans (reviewed in [6]). nAChRs - in particular, the $\alpha 7 \mathrm{nAChR}$ - may also be involved in amyloid beta-induced neurotoxicity. The US FDA-approved drug galantamine is a putative allosteric activator of nAChRs in addition to its AChE-inhibitory profile, and therefore may actually be neuroprotective and slow disease progression in patients $[7,8]$.

\section{M1 Muscarinic acetylcholine receptors}

In addition to nicotinic agonists, M1 muscarinic agonists have the potential to boost cognition and slow disease progression. Postsynaptic M1 muscarinic acetylcholine receptors play a major role in hippocampal-based memory and learning. These receptors are relatively spared from degeneration in $\mathrm{AD}$, so even when $\mathrm{AChE}$ inhibitors lose their efficacy because the disease has caused presynaptic degeneration and decreasing acetylcholine levels, MI selective muscarinic agonists may continue to produce a cognitive benefit, although specificity is critical. In addition, M1 muscarinic agonists also have the potential to affect multiple disease pathologies including amyloid-beta and phosphorylated tau through regulation of a-disintegrin and metalloprotease 10/17 and of glycogen synthase kinase $3 \beta$ activity, respectively (reviewed in [9]).

\section{5-Hydroxytryptamine receptors}

In contrast to direct cholinergic stimulation, another potential therapeutic avenue is indirect cholinergic stimulation through 5-hydroxytryptamine $(5-\mathrm{HT})_{6}$ receptors. Evidence from cellular and animal models suggests that $5-\mathrm{HT}_{6}$ receptors may enhance cholinergic signaling through glutamatergic and gamma-aminobutyric acidergic pathways (reviewed in [10]). Both agonist and antagonist ligands of this poorly understood G-protein -coupled receptor have been shown to enhance cognition in preclinical rodent models. These findings may be explained by differential receptor coupling to multiple $G_{\alpha}$ subunits or by the potential for agonist and antagonist $5-\mathrm{HT}_{6}$ ligands to act on distinct neuronal populations. Several ligands currently in early-phase clinical trials show promise as symptomatic treatments for $\mathrm{AD}$ and are also being evaluated in combination with $\mathrm{AChE}$ inhibitors. Of note, Lundbeck's 5- $\mathrm{HT}_{6}$ receptor antagonist Lu AE58054 met its primary endpoint in a recent clinical study [11].

\section{Norepinephrine}

Modulation of the noradrenergic system has the potential to be both a symptomatic and disease-modifying therapeutic strategy. The noradrenergic neurons of the locus coeruleus exhibit selective vulnerability during aging, with a significant loss of these cells and a decrease in norepinephrine observed in mild cognitive impairment and AD patients (reviewed in [12]). Norepinephrine is an excitatory neurotransmitter that also has anti-inflammatory properties. Regulators of norepinephrine levels are clinically approved for use in neuropsychiatric disorders and have demonstrated positive symptomatic and disease-modifying effects in preclinical AD studies [13]. In clinical studies, the norepinephrine reuptake inhibitor atomoxetine improved overall global cognition in a small study of Parkinson's disease patients. A phase IIa study in amnestic mild cognitive impairment patients is currently being led by Allan Levey (Emory University) and colleagues to determine the effects of atomoxetine on biomarkers of $\mathrm{AD}$ pathology, inflammation and cognitive function (ClinicalTrials.gov: NCT01522404). This provides an exciting opportunity for repurposing of a US FDA-approved drug, which if successful will accelerate the drug's availability for AD patients.

\section{Cyclic adenosine monophosphate}

Although the hippocampus is the brain area most frequently discussed in relation to $\mathrm{AD}$, the prefrontal cortex is critical for higher-level cognitive function and is sensitive to aging (reviewed in [14]). Within this brain area, cAMP signaling is increased with aging leading to an increased opening of hyperpolarization-activated cyclic nucleotide-gated potassium channels and a reduced activity of neurons involved with working memory. This cAMP prefrontal cortex pathway has been successfully targeted to improve cognitive function in attention-deficit hyperactivity disorder and other disorders using guanfacine, an alpha-2A adrenergic receptor agonist that was originally approved by the US FDA for anti-hypertensive effects. The ability of guanfacine to enhance cognitive function in older people with cognitive aging has recently been tested in a clinical trial by Christopher Van Dyck, based in large part on research by Amy Arnsten and others at Yale University [15].

\section{Conclusion}

There is currently lack of consensus in the field on the neurochemical and pathological differences between cognitive aging and $\mathrm{AD}$. We do not yet know whether cognitive aging is a discrete clinical syndrome or simply an earlier stage in the continuum of AD. Many of the therapeutic approaches described above as well as other cognitive enhancer strategies (including inhibitors of phosphodiesterases, monoamine oxidase B, histamine $\mathrm{H} 3$ receptor, histone deacetylases, among others) target general mechanisms associated with learning and memory and would probably benefit individuals suffering from both cognitive aging and AD. Novel cognitive-enhancing therapeutics will also be useful as 
investigational tools to further explore the mechanistic differences between normal cognitive aging and AD.

As these cognitive-enhancing therapeutics enter clinical testing, the need for quantitative and sensitive biomarkers to track cognitive function for both patient selection and for monitoring therapeutic response is critical. Combining cognitive testing paradigms with physical biomarkers such as functional imaging and electroencephalography may provide greater insight into underlying disease mechanisms and increase the potential effectiveness of these trials. These biomarkers will also help to elucidate differences or similarities between normal cognitive aging and AD. Ultimately, a greater understanding of the pathobiology of cognitive dysfunction with aging will accelerate the development of effective cognitive enhancers for both cognitive aging and AD.

Note: This article is part of a series on Traumatic Brain Injury, edited

by Robert Stern. Other articles in this series can be found at

http://alzres.com/series/traumaticbraininjury.

\section{Abbreviations}

AChE: Acetylcholinesterase; AD: Alzheimer's disease; FDA: Food and Drug Administration; 5-HT: 5-hydroxytryptamine; mGlu5: Metabotropic glutamate receptor subtype 5; nAChR: Neuronal nicotinic acetylcholine receptor.

\section{Competing interests}

The authors declare that they have no competing interests.

Published: 02 Oct 2013

\section{References}

1. Nehlig A: Is caffeine a cognitive enhancer? J Alzheimers Dis 2010, 20(Suppl 1):S85-S94

2. Nachum-Biala Y, Troen AM: B-vitamins for neuroprotection: narrowing the evidence gap. Biofactors 2012, 38:145-150.

3. Gomez-Pinilla F: Collaborative effects of diet and exercise on cognitive enhancement. Nutr Health 2011, 20:165-169.

4. Chiesa A, Calati R, Serretti A: Does mindfulness training improve cognitive abilities? a systematic review of neuropsychological findings. Clin Psychol Rev 2011, 31:449-464.

5. Noetzel MJ, Gregory K, Vinson PN, Manka JT, Stauffer SR, Lindsley CW, Niswender CM, Xiang Z, Conn PJ: A novel metabotropic glutamate receptor 5 positive allosteric modulator acts at a unique site and confers stimulus bias to mGlu5 signaling. Mol Pharmacol 2013, 83:835-847.

6. Wallace TL, Porter RH: Targeting the nicotinic alpha7 acetylcholine receptor to enhance cognition in disease. Biochem Pharmacol 2011, 82:891-903.

7. Engedal K, Davis B, Richarz U, Han J, Schauble B, Andreasen N: Two galantamine titration regimens in patients switched from donepezil. Acta Neurol Scand 2012, 126:37-44.

8. Geerts H: Indicators of neuroprotection with galantamine. Brain Res Bull 2005, 64:519-524.

9. Fisher A: Cholinergic treatments with emphasis on $\mathrm{m} 1$ muscarinic agonists as potential disease-modifying agents for Alzheimer's disease. Neurotherapeutics 2008, 5:433-442.

10. Dawson LA: The central role of 5-HT6 receptors in modulating brain neurochemistry. Int Rev Neurobiol 2011, 96:1-26.

11. Phase II clinical data show statistically significant improvement for Lu AE58054 as addon to donepezil, versus donepezil alone, on cognitive symptoms of Alzheimer's disease. http://investor.lundbeck.com/ releasedetail.cfm?releaseid $=777646$.
12. Chalermpalanupap T, Kinkead B, Hu WT, Kummer MP, Hammerschmidt T, Heneka MT, Weinshenker D, Levey Al: Targeting norepinephrine in mild cognitive impairment and Alzheimer's disease. Alzheimers Res Ther 2013, 5:21.

13. Kalinin S, Polak PE, Lin SX, Sakharkar AJ, Pandey SC, Feinstein DL: The noradrenaline precursor L-DOPS reduces pathology in a mouse model of Alzheimer's disease. Neurobiol Aging 2012, 33:1651-1663.

14. Samson RD, Barnes CA: Impact of aging brain circuits on cognition. Eur J Neurosci 2013, 37:1903-1915.

15. Arnsten AF, Jin LE: Guanfacine for the treatment of cognitive disorders: a century of discoveries at Yale. Yale J Biol Med 2012, 85:45-58.

\subsection{6/alzrt209}

Cite this article as: Shineman et al:: Progress in novel cognitive enhancers for cognitive aging and Alzheimer's disease. Alzheimer's Research \& Therapy 2013, 5:45 